\title{
Concomitant bilateral intravitreal anti-VEGF injections for the treatment of exudative age-related macular degeneration
}

This article was published in the following Dove Press journal:

Clinical Ophthalmology

25 June 2010

Number of times this article has been viewed

R Prince Davis

Amy C Schefler

Timothy G Murray

Bascom Palmer Eye Institute, Department of Ophthalmology, University of Miami Miller School of Medicine, Miami, FL, USA
Correspondence:Timothy G Murray Bascom Palmer Eye Institute, 900 NW 17th Street, Miami, FL 33।36, USA

$\mathrm{Tel}+\mathrm{I} 3053266166$

Fax + I 3055473713

Email tmurray@med.miami.edu
Purpose: To report the outcomes of same-day, bilateral intravitreal anti-vascular endothelial growth factor (VEGF) therapy for a consecutive series of patients treated for exudative age-related macular degeneration (ARMD).

Methods: A consecutive series of 254 eyes of 127 patients received bilateral, same-day anti-VEGF injections of either bevacizumab or ranibizumab between January 1, 2007 and July 1, 2008 and the outcomes were assessed. Approval was obtained from the Institutional Review Board at the University of Miami Miller School of Medicine.

Results: Bilateral, same-day anti-VEGF injections were well tolerated in all patients receiving the therapy. Indication for injection was exudative macular degeneration in all patients. The incidence of adverse effects was low, with only four events reported, none of which were serious.

Conclusions: In this study, bilateral anti-VEGF injections performed on the same day were preferred over staggered injections and were well tolerated by patients. No major systemic or ocular adverse events were noted, despite the presence of risk factors in a subset of patients. Given that there are serious, albeit rare, complications following anti-VEGF injection, further study with a larger number of patients will be necessary to definitively prove the safety of this treatment modality.

Keywords: visual acuity, same-day treatment, bevacizumab, ranibizumab

\section{Introduction}

Vascular endothelial growth factor (VEGF) is increasingly recognized as an important etiologic agent in several ophthalmic disease processes. Besides its antineoplastic properties, anti-VEGF has been shown to be effective in the treatment of various eye conditions such as exudative age-related macular degeneration (ARMD), diabetic macular edema, and retinal vein occlusions. ${ }^{1-3}$ Because of their proven efficacy and safety profiles, anti-VEGF agents are increasingly being used to augment or even supplant other therapeutic modalities.

Not infrequently, multiple injections are required to treat exudative ARMD. A patient with active bilateral disease may require twice-monthly visits to his/her treating ophthalmologist for an extended period of time, assuming that the bilateral injections are given in a staggered fashion. This frequency of treatment would pose a significant burden on patients' time and productivity, and it increases the administrative load on the health care system.

Prior to the advent of anti-VEGF therapy, many intravitreal injections in patients with bilateral ocular disease were of triamcinolone. As intravitreal triamcinolone (IVTA) is opaque and has a significant incidence of transient visual symptoms that 
may last for hour or days, bilateral, same-day injections are usually contraindicated owing to the possibility that both eyes may simultaneously experience vision-limiting media opacity immediately following injection. The anti-VEGF agents, such as ranibizumab (rhuFab V2; Lucentis ${ }^{\circledR}$, Genentech, South San Francisco, CA, USA) and bevacizumab (Avastin ${ }^{\circledR}$, Genentech, South San Francisco, CA, USA) have proven to have an acceptable safety profile with low rates of cataract formation, elevated intra-ocular pressure (IOP), endophthalmitis, and retinal detachment. ${ }^{4,5}$ As these medications are transparent, transient visual reduction due to media opacity is typically less pronounced than with triamcinolone. The theoretical risk of functional visual decline from bilateral, same-day anti-VEGF injection is therefore quite low. In this article, we report on our experience with patients treated with bilateral, same-day anti-VEGF therapy.

\section{Methods}

Cases consisted of 254 eyes from 127 consecutive patients given bilateral, same-day injections of either ranibizumab or bevacizumab between January 1, 2007 and July 1, 2008. All patients were under the care of a single ophthalmologist (TGM) at the Bascom Palmer Eye Institute (Miami, FL, USA). One patient was excluded from analysis as she was lost to follow up.

Pre-injection clinical features recorded were age, indication for injection, drug injected, concentration and volume of injection, and Snellen visual acuity (VA). Post-injection visual acuity, adverse events, and need for additional injection were also examined. All patients underwent examination with Stratus optical coherence tomography (Stratus $\mathrm{OCT}^{\mathrm{TM}}$ ) (Carl Zeiss Meditec, Inc.; Dublin, CA, USA) immediately prior to injection and at each follow-up visit. Pre- and post-injection central retinal thicknesses were recorded. Presence or absence of a pigment epithelial detachment (PED) was also recorded.

This study was approved by the Institutional Review Board of the University Of Miami, Miller School of Medicine. Informed consent was obtained from each patient prior to injection. In patients receiving bevacizumab, the off-label use of the medication was discussed with the patient in detail. All patients studied received either $1.25 \mathrm{mg} / 0.05 \mathrm{~mL}$ of bevacizumab or $0.5 \mathrm{mg} / 0.05 \mathrm{~mL}$ of ranibizumab injected intravitreally through the pars plana in each eye. Each eye was prepared in a sterile manner and injected separately. The preparation was the standard pre-injection protocol for the Bascom Palmer Eye Institute, which includes 10\% povidone-iodine to the periorbital skin and lashes followed by insertion of a sterile eyelid speculum and alternating instillation of three rounds of 5\% povidone-iodine and three rounds of a sterile aliquot of $4 \%$ lidocaine to the inferior fornix and conjunctiva at the injection site. No drops or equipment were reused in the preparation of the second eye. Post-injection, each patient was placed on a treatment of topical fourth-generation fluoroquinolone 4 times per day for 4 days.

\section{Results}

A total of 1322 injections was performed on 254 eyes from 127 patients (43 men, 84 women). Mean patient age was 82.5 years $($ median $=83$, range $=56-101)$. The length of the follow-up period was 1-26 months, with a mean and median follow-up period of 14.2 months and 15.3 months, respectively. Indication for treatment was bilateral exudative ARMD in all patients. Of the patients studied, 32 had never had an intravitreal anti-VEGF injection before, 57 had a history of unilateral anti-VEGF injection, and 38 had a history of bilateral anti-VEGF injections given in a staggered fashion (3-10 days between injections). One patient was excluded from analysis due to lack of follow-up examination data.

Twenty-two patients (17\%) were on oral anticoagulation therapy with warfarin or clopidogrel at the time of injection; one patient (1\%) was using aspirin/dipyridamole; and 31 patients $(24 \%)$ were taking aspirin (one of these patients was on both aspirin and warfarin). Eighty-one patients (60\%) had a history of hypertension; four patients (3\%) had had an ischemic stroke; 17 patients $(13 \%)$ had coronary artery disease, 6 of whom $(5 \%)$ had had a myocardial infarction. Three patients $(2 \%)$ had a unilateral pigment epithelial detachment (PED), and one patient (1\%) had bilateral pigment epithelial detachments at the time of first bilateral injection.

Mean Snellen VA, pre-injection, was 20/175 in the better eye $(n=127)$ and $20 / 1175$ in the worse eye $(n=127)$. Mean pre-injection central retinal thickness (CRT), as determined by OCT, was 238 microns $(n=251)$. One month after the first bilateral injection, mean VA was 20/144 $(n=116)$ in the better eye and 20/1050 $(n=116)$ in the worse eye; mean CRT was $235(n=232)$. Of the 119 patients with at least 3 months of follow-up, mean visual acuity at 3 months post-injection was 20/127 $(n=119)$ in the better eye and 20/973 $(n=119)$ in the worse eye (the mean pre-injection visual acuity for this subset of patients was 20/175 in the better eye and 20/1020 in the worse eye). Mean CRT was $248(n=138)$ at 3 months after the first injection.

A mean of 5.2 bilateral injections was performed per patient. 107 patients (84\%) required bilateral reinjection 
during the follow-up period, and 13 patients (10\%) required subsequent unilateral injection. The mean interval to second injection was 1.3 months (median $=1$ month, range $=3$ weeks -5 months). Seven patients required no subsequent injections in either eye. Indication for reinjection was any residual or new intraretinal and/or subretinal fluid, found via OCT analysis.

Adverse events following injection were recorded after 4 injections. Three patients reported pain and/or tearing following injection and 1 patient had an episode of supraventricular tachycardia requiring transfer to a local hospital. No eyes developed endophthalmitis, anterior chamber cell or flare, vitritis, vitreous hemorrhage, retinal detachment, or retinal pigment epithelial tear. Eight patients died during the followup period as identified by the Social Security Death Index (SSDI). No patients had a myocardial infarction or stroke.

\section{Discussion and conclusions}

The safety and efficacy of bevacizumab and ranibizumab have been shown in several studies. ${ }^{1-5}$ Outcomes from bilateral, same-day injections of these agents, however, have only recently begun to be elucidated. This retrospective study presents early data showing that bilateral, same-day injections are well received by patients and seem to have minimal adverse effects in the limited number of patients studied.

Prior to the advent of the anti-VEGF agents, bilateral, same-day intravitreal injections were usually performed only in patients with sight-threatening disease. There is a long history of bilateral injection of intravitreal antibiotics and antivirals for conditions such as viral retinitis and endogenous endophthalmitis. Efficient study of the safety and tolerability of such injections, however, is precluded by a high instance of disease-related complications. With bevacizumab and ranibizumab, some of the recognized serious adverse events following intravitreal injection include retinal pigment epithelial tear, retinal detachment, endophthalmitis, vitreous hemorrhage, and a possible increase in stroke risk. ${ }^{1,4,6,7}$ In our study, none of these serious adverse events occurred in any patient; however, larger numbers of patients will need to be studied to ensure there is no significant increase in the risk of these complications.

While an interim analysis of the SAILOR (safety assessment of intravitreal Lucentis for AMD) data have suggested a statistically significant increase in stroke risk in patients receiving $0.5 \mathrm{mg}$ of intravitreal ranibizumab when compared to those receiving a lower, $0.3 \mathrm{mg}$, dose, the MARINA (minimally classic/occult trial of the anti-VEGF antibody ranibizumab in the treatment of neovascular AMD) and ANCHOR (Anti-VEGF antibody for the treatment of predominantly classic choroidal neovascularization (CNV) in AMD) studies revealed no statistically significant difference. ${ }^{1,8}$ Similar analysis of pharmacokinetic data for rabbits has shown that the serum concentration of ranibizumab following intravitreal injection is 1500 fold lower than the intravitreal concentration. ${ }^{9}$ The role of VEGF in stroke is still unclear and further prospective study is required to elucidate the possible association of anti-VEGF agents with stroke. In this study, no ischemic strokes were reported. Given the uncertainty of stroke risk with these agents, it may be wise to consider staggered therapy in patients with strong risk factors for ischemic stroke (history of stroke/ischemia, uncontrolled hypertension, etc.).

Given the association of the anti-VEGF agents with retinal pigment epithelial (RPE) tear, it is possible that bilateral, same-day injections may result in bilateral occurrence of such an event. Only one case report of bilateral RPE tear was found in our literature search; Norose et al reported a case of an RPE tear following argon laser photocoagulation of a $4 \times 6$-disc-diameter pigment epithelial detachment (PED) in the patient's right eye followed by a spontaneous RPE tear along the margin of a 1-disc-diameter PED in the untreated fellow eye 5 days later. ${ }^{10}$ No bilateral RPE tears have been reported with anti-VEGF therapy. However, Chang and Sarraf reviewed all published cases of RPE tear after anti-VEGF therapy and showed that all RPE tears associated with this therapy occurred in the setting of exudative ARMD, specifically in patients with fibrovascular PED or PED associated with choroidal neovascularization. ${ }^{6}$ Kook et al reported the incidence of RPE tear in patients with exudative ARMD and PED that had been treated with bevacizumab to be $18 \%{ }^{11}$ Given this high incidence, administering bilateral, same-day injections may warrant caution in patients with bilateral PED. It is worth noting, however, that Chang and Sarraf showed that the mean time between anti-VEGF injection and diagnosis of RPE tear is 4.5 weeks, ${ }^{6}$ suggesting that concomitant bilateral injections may pose no greater risk than would a staggered strategy.

With IVTA, post-injection inflammation can lead to decline of visual acuity and can even mimic endophthalmitis in some cases. ${ }^{12}$ Uveitis and vitritis have been reported following injection of anti-VEGF agents, but the incidence of this side effect appears to be low. ${ }^{4,13}$ Kiss et al prospectively studied 61 patients that received intravitreal bevacizumab with a laser flare meter and showed that no patient had any significant anterior segment inflammatory response in the follow-up interval. ${ }^{14}$ Similarly, Spaide et al examined 266 eyes 
of 266 patients that had received intravitreal bevacizumab for CNV secondary to ARMD and found that a mild vitritis was present in three patients. ${ }^{13}$ The vitritis, however, did not present until 1 month post-injection in two patients and 2 months post-injection in the third patient. While an argument can be made that bilateral, same-day injection may lead to bilateral vitritis or pseudoendophthalmitis, the data from Spaide et al suggest that the onset may be delayed enough that even patients receiving staggered injections may have both eyes injected before either eye develops an inflammatory response. Looking at the experience with IVTA, Marticorena et al reported a patient presenting with recurrent uveitis and vitritis following multiple sessions of IVTA and photodynamic therapy (PDT). ${ }^{15}$ This report and others suggest a potential etiology of hypersensitivity to the drug or vehicle. Although post-injection inflammation appears to be lower with anti-VEGF agents than with IVTA, clinical experience with these agents is limited. It is therefore reasonable to consider a unilateral trial of intravitreal anti-VEGF therapy in non-emergent patients with no injection history to establish that the drug is well tolerated.

Another concern with injection of any intravitreal agent bilaterally is the possibility of simultaneous intraocular hemorrhages in both eyes. While subconjunctival hemorrhage is often seen following injection, it is rarely visually significant. Submacular and vitreous hemorrhage, however, are of concern given the potential for a large decrease in visual function for a prolonged period of time. Chieh et al have reported a case of submacular hemorrhage that occurred 4 weeks after bevacizumab therapy for occult $\mathrm{CNV}$ in ARMD. ${ }^{16}$ Similarly, Cleary et al assessed the effects of intravitreal injections of bevacizumab in 112 eyes and found three instances of submacular hemorrhage. ${ }^{17} \mathrm{Wu}$ et al had one episode of vitreous hemorrhage in their study of 4303 intravitreal bevacizumab injections in 1310 consecutive eyes. ${ }^{4}$ The rates of submacular hemorrhage and vitreous hemorrhage appear to be quite low; however, the onset of bilateral intraocular hemorrhage has the potential to be visually devastating. As bevacizumab given intravenously for cancer treatment has been shown to increase the risk of both bleeding and thrombosis, ${ }^{18}$ the risk of vitreous hemorrhage may be increased in patients receiving intravitreal anti-VEGF. Although there are no data on the risk profile of patients receiving intravitreal anti-VEGF therapy that are also on anticoagulation therapy, it is plausible that such patients may be at increased risk for intraocular bleeding events. As mentioned above, in our study, $23 \%$ of the patients were on aspirin and 19\% were taking the anticoagulants warfarin, clopidogrel, or aspirin/dipyridamole, and no patients experienced any intraocular hemorrhage.

While this study did not formally address the question of satisfaction after bilateral injections, it has been our experience that patients with bilateral AMD prefer concomitant, same-day injections over staggered injections. In the subset of our patients that had previously undergone staggered bilateral injection, many have had multiple sets of bilateral, same-day injections and very few have switched back to the staggered injections. Further prospective study of all patients offered bilateral, same-day injections, involving a formal satisfaction questionnaire, would be helpful in objectively assessing patient preference.

A transient decline in vision after intravitreal injections has not been described in the literature; however, our anecdotal experience has shown a widely variable range of visual decline secondary to toxicity from the preparation or post-injection media opacity from the agent itself that may last for several days. We therefore utilized a treatment strategy of staggered injections in patients requiring bilateral therapy with IVTA. As bevacizumab and ranibizumab are both transparent, media opacity is much less of a concern in patients treated with these agents. The index of refraction of bevacizumab and ranibizumab is essentially equivalent to that of $0.9 \%$ normal saline at 1.33 , whereas the index of refraction of the normal adult vitreous fluid is 1.337. This slight difference may be enough to cause transient visual symptoms (floaters, mild distortion) following injection, but is likely not great enough to significantly affect visual acuity. An interesting avenue of further study would be to assess visual acuity immediately after anti-VEGF injection and compare it with that following IVTA and sham injection. In addition to changes in visual acuity induced by the intravitreal agent, there may be a significant effect on vision from the topical application of lidocaine or povidone-iodine solutions onto the cornea, corneal surface changes from lid speculum-induced dry eye, and corneal epithelial alteration resulting from the decreased blink response after topical anesthesia. As many patients who require anti-VEGF therapy often have visual acuity that limits their ability to read, work, or drive legally, quantification of the visual effects of sterile preparation and injection of various intravitreal agents would be helpful in post-injection functional counseling.

In summary, because bevacizumab and ranibizumab are both transparent drugs with minimal theoretical risk of transient visual decline, concomitant bilateral injections may be used in appropriate patients. In our study, we noted no serious adverse effects from such a treatment strategy despite the presence 
of significant risk factors. Care should be taken with patients currently on anticoagulation therapy, with those having bilateral PED, and with patients having significant stroke risk factors, as these patients may experience simultaneous onset of systemic or bilateral ocular adverse effects that could limit their visual acuity. Adopting a staggered injection strategy in such patients might reduce the risk of bilateral complications that may significantly limit a patient's ability to function in his/her activities of daily living.

Bilateral injections of ranibizumab and bevacizumab are well tolerated and in many instances are preferred by patients with a prior history of staggered injections. When compared to staggered injections, a bilateral, same-day injection strategy is beneficial in that it decreases the administrative load on the health care system (eg, fewer appointments, lower number of insurance claims to submit and process), reduces the amount of time patients spend in the office, and frees up appointment slots for other patients.

\section{Disclosure}

The authors report no conflicts of interest in this work.

\section{References}

1. Rosenfeld PJ, Brown DM, Heier JS, Boyer DS, Kaiser PK, Chung CY, Kim RY: MARINA Study Group. Ranibizumab for neovascular age-related macular degeneration. $N$ Engl J Med. 2006;355(14): 1419-1431.

2. Priglinger SG, Wolf AH, Kreutzer TC, Kook D, Hofer A, Strauss RW, Alge CS, Kunze C, Haritoglou C, Kampik A. Intravitreal vebacizumab injections for treatment of central retinal vein occlusion: six month results of a prospective trial. Retina. 2007;27(8):1004-1012.

3. Arevalo JF, Fromow-Guerra J, Quiroz-Mercado H, et al. Primary intravitreal bevacizumab (Avastin) for diabetic macular edema: results from the Pan-American Collaborative Retina Study Group at 6-month follow-up. Ophthalmology. 2007;114(4):743-750.
4. Wu L, Martinez-Castellanos MA, Quiroz-Mercado H, et al. for the Pan American Collaborative Retina Group (PACORES). Twelve-month safety of intravitreal injections of bevacizumab (Avastin ${ }^{\circledR}$ ): results of the Pan-American Retina Study Group (PACORES). Graefes Arch Clin Exp Ophthalmol. 2008;246(1):81-87.

5. Rosenfeld PJ, Rich RM, Lalwani GA. Ranibizumab. Phase III clinical trial results. Ophthalmol Clin North Am. 2006;19(3):361-372.

6. Chang LK, Sarraf D. Tears of the retinal pigment epithelium: an old problem in a new era. Retina. 2007;27(5):523-534.

7. Ronan SM, Yoganathan P, Chien FY, et al. Retinal pigment epithelium tears after intravitreal injection of bevacizumab (Avastin) for neovascular age-related macular degeneration. Retina. 2007;27(5):535-540.

8. Brown DM, Kaiser PK, Michels M, et al. Ranibizumab versus verteporfin for neovascular age-related macular degeneration. $N$ Engl J Med. 2006;355(14):1432-1444.

9. Gaudreault J, Fei D, Rusit J, Suboc P, Shiu V. Preclinical pharmacokinetics of ranibizumab (rhuFabV2) after a single intravitreal administration. Invest Ophthalmol Vis Sci. 2005;46(2):726-733.

10. Norose K, Tanino T, Segawa K. Bilateral tears of the retinal pigment epithelium. Br J Ophthalmol. 1988;72(8):621-627.

11. Kook D, Wolf A, Neubauer AS, et al. Retinal pigment epithelial tears after intravitreal injection of bevacizumab for AMD: frequency and progress [German]. Ophthalmologe. 2008;105(2):158-164.

12. Ozkiriş A, Erkiliç K. Complications of intravitreal injection of triamcinolone acetonide. Can J Ophthalmol. 2005;40(1):63-68.

13. Spaide RF, Laud K, Fine HF, et al. Intravitreal bevacizumab treatment of choroidal neovascularization secondary to age-related macular degeneration. Retina. 2006;26(4):383-390.

14. Kiss C, Michels S, Prager F, Weigert G, Geitzenauer W, SchmidtErfurth U. Evaluation of anterior chamber inflammatory activity in eyes treated with intravitreal bevacizumab. Retina. 2006;26(8):877-881.

15. Marticorena J, Gomez-Ulla F, Romano MR, Luna I. Repeated psuedoendophthalmitis after combined photodynamic therapy and intravitreal triamcinolone. Graefes Arch Clin Exp Ophthalmol. 2007;245(9):1403-1404.

16. Chieh JJ, Fekrat S. Large subretinal hemorrhage after intravitreal bevacizumab (Avastin) for age-related macular degeneration. Ann Ophthalmol (Skokie). 2007;39(1)51-52.

17. Cleary CA, Jungkim S, Ravikumar K, Kelliher C, Acheson RW, Hickey-Dwyer M. Intravitreal bevacizumab in the treatment of neovascular age-related macular degeneration, 6- and 9-month results. Eye. 2008;22(1)82-86.

18. Kamba T, McDonald DM. Mechanisms of adverse effects of anti-VEGF therapy for cancer. Br J Cancer. 2007;96(12):1788-1795.
Clinical Ophthalmology

\section{Publish your work in this journal}

Clinical Ophthalmology is an international, peer-reviewed journal covering all subspecialties within ophthalmology. Key topics include: Optometry; Visual science; Pharmacology and drug therapy in eye diseases; Basic Sciences; Primary and Secondary eye care; Patient Safety and Quality of Care Improvements. This journal is indexed on

Submit your manuscript here: http://www.dovepress.com/clinical-ophthalmology-journal

\section{Dovepress}

PubMed Central and CAS, and is the official journal of The Society of Clinical Ophthalmology (SCO). The manuscript management system is completely online and includes a very quick and fair peer-review system, which is all easy to use. Visit http://www.dovepress.com/ testimonials.php to read real quotes from published authors. 